\title{
Overlapped k-Space Acquisition and Reconstruction Technique for Motion Artifact Reduction in Magnetic Resonance Imaging
}

\author{
Yasser M. Kadah \\ Biomedical Engineering Department, Cairo University, Giza 12613, Egypt
}

\begin{abstract}
A new MRI acquisition strategy based on acquiring the k-space in consecutive overlapped bands was developed. Starting from the general assumption of rigid body motion, we consider the case when the acquisition of the $\mathrm{k}$-space is in the form of bands of finite number of lines arranged in a rectilinear fashion to cover the k-space area of interest. We consider cases with an averaging factor of at least 2. Instead of acquiring a full k-space of each image and then average the result, we developed a new acquisition strategy based on acquiring the k-space in consecutive overlapped bands. In case of no motion, this overlap can be used as the second acquisition. On the other hand, when motion is encountered, such overlap can be used to substantially reduce motion artifacts in the resultant image. This is achieved by utilizing the overlap area to estimate the motion, which is then taken into consideration in image reconstruction. We demonstrate the success of this approach using both numerical simulations as well as real data acquired from a human volunteer. The new method has the potential for practical applications to make more efficient use of MRI scanners and making the scanning time lower providing more comfort to the patient.
\end{abstract}

Keywords: Motion Artifact, Magnetic Resonance Imaging, Artifact Suppression, Navigator Echo.

\section{INTRODUCTION}

Accurate diagnosis in medical procedures has become widely attainable by the advent of the different medical imaging modalities. Among those, magnetic resonance imaging (MRI) is currently one of the most promising non-invasive diagnostic tools in medicine. In addition to its ability to produce anatomical images of remarkable detail and contrast, it can be used to visualize vascular structures, measure blood flow and perfusion, detect neural activation, and identify the metabolic information of different areas in the acquired images. Also, its inherently volumetric acquisition permits slices at different angles to be computed easily, which can be advantageous in many applications.

One of the major problems with the present MRI technology is its susceptibility to substantial artifacts when motion occurs during image acquisition. Even though, fast acquisition methods, such as EPI and spiral imaging, provide a solution to this problem for some applications, these techniques are extremely sensitive to magnetic field inhomogeneity as compared to regular scanning methods and have a generally low signal-to-noise ratio. This makes it difficult to accurately correlate the generated images with the physical anatomy because of geometric distortion in addition to more profound signal loss within the areas of large susceptibility mismatches. Moreover, when these imaging sequences are used in applications such as functional magnetic resonance imaging (fMRI), where a set of slices are acquired repeatedly, patient motion persists in the form of low detection of activation sites as a result of misregistration of images along the sequences. ${ }^{1}$

Due to practical constraints from the MRI machine hardware, signal-to-noise ratio, and image contrast of MRI, the imaging time commonly extends to several minutes. As a result, different parts of the collected k-space are acquired at different time instants. In an ideal scenario, the imaged object does not change during the period of the experiment, and the image calculated by inverse Fourier transformation is undistorted. However, in clinical MRI setups, this scenario is usually not guaranteed, because of physiological and occasional voluntary patient motion and can be even impossible to realize for moving organs such as the heart and abdominal structures. Consequently, the constructed images suffer varying degrees of distortion depending on the characteristics of the imaging sequence and the severity of motion during the scan duration.

Motion artifacts can generally be classified into either intraslice or inter-slice motion. ${ }^{1-2}$ The first is the result of motion in between the acquisition of different portions of the k-space while the second is the result of motion in between acquisitions of the same slice. An illustration of these types of motion is shown in Figure 1. The techniques in the literature often treated 

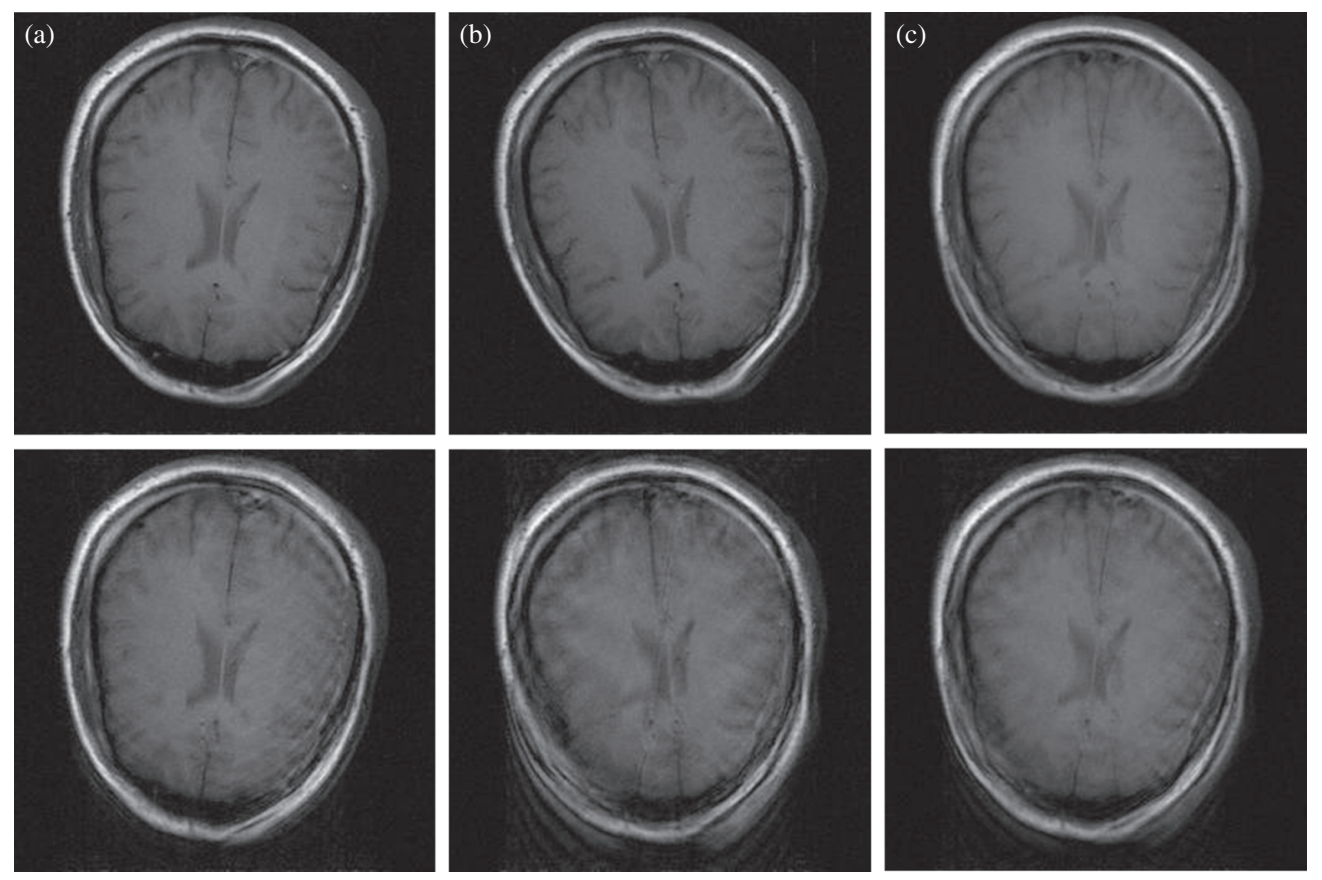

Fig. 1. Examples of motion artifacts: inter-slice motion artifact (above) results when images (a) and (b) are averaged to create the final image (c) on the system. On the other hand, if the same process is repeated with these individual images having an intra-slice motion (below), the result will be a more severe combined intra- and inter-slice motion artifact on image (c) below.

these types in completely different manners with several strategies to suppress each type independently. Given their underlying similarities, it might be advantageous to treat both problems simultaneously.

Several attempts to solve the problem of intraslice motion artifact in MRI have been reported in the literature. In general, the available techniques can be classified into four main categories. The first category attempts to suppress relative patient motion among different $\mathrm{k}$-space lines within a given image through either through breath holding and chest strapping or by using cardiac and respiratory gating. ${ }^{7}$ This minimizes the physiological component of motion between these lines at the expense of increased discomfort to the patient and/or significantly longer acquisition times. The second category uses averaging of different acquisitions to suppress the motion artifacts as well as to improve the signal-to-noise ratio of the final image. This can be done by taking the average of the corresponding k-space lines in a number of consecutive image acquisitions, or more generally by composing a weighted average of the two based on optimizing a certain objective function under given constraints. ${ }^{7,18}$ The third category applies extra magnetic gradient lobes in the imaging sequence to eliminate the effects of motion through signal refocusing assuming a simple polynomial model for this motion. ${ }^{20,23}$ This technique is used to minimize signal loss from moving blood and CSF within a given voxel ${ }^{17}$ Finally, the fourth category assumes simple forms of rigid body motion including translational and rotational components and corrects for them in a post-processing step. The motion in this category is estimated using external monitoring, ${ }^{24}$ navigator echo, ${ }^{1,2}$ symmetry constraints, ${ }^{13}$ motion periodicity constraint,,${ }^{8,16}$ or through automated estimation using different measures in postprocessing techniques. $6,9,10,14,15,19,21,22,25$ The effect of translational motion can be suppressed by post-processing through modifying the phase of the k-space lines according to the a priori knowledge about the motion. ${ }^{11,12,21}$ In practice, the navigator echo techniques are the most useful when they can be incorporated into the imaging sequence of interest. On the other hand, postprocessing techniques are not as useful in practice given their very high computational complexity and convergence problems. The technique in Ref. [6] and its modification in Ref. [25] provide the best results in this category.

The inter-slice motion problem is a significantly simpler problem whereas rigid-body registration between consecutive images is sufficient for practical purposes. Among the several available techniques to estimate and correct for such motion, the Automated Image Registration (AIR) technique ${ }^{26}$ is widely used in MRI applications (such as functional MRI image registration) as well as in multimodal image registration.

In this work, we propose a new approach for suppressing motion artifacts from both types. The proposed method assumes rigid body motion and corrects for both its translational and rotational motion components without need for extra acquisitions. The new method is an extension of an earlier work done by our group ${ }^{2,3}$ whereby the floating navigator is generalized to include multiple lines acquired within the regular data acquisition and their data are registered using a similar technique as the AIR technique in the k-space. The new method is verified using numerical simulations as well as real data from a normal human volunteer.

\section{METHODOLOGY}

We consider the case of rigid body motion, which is widely encountered in magnetic resonance images of the brain and limbs. The relation between the MR signal and the $2 \mathrm{D}$ density distribution of the target in the imaging plane is given by, 


$$
F\left(k_{x}, k_{y}\right)=\int_{-\infty}^{\infty} \int_{-\infty}^{\infty} f(x, y) \exp \left[-j 2 \pi\left(k_{x} x+k_{y} y\right)\right] d x d y
$$

where $F\left(k_{x}, k_{y}\right)$ is the MR signal, $k_{x}$ and $k_{y}$ are the spatial frequency coordinates in the readout and phase-encoding directions, respectively, $f(x, y)$ is the density distribution of the nonmoving imaging target, and $x, y$ are horizontal and vertical coordinates in the imaging plane. In (1) it is seen that the MRI signal is the 2-D Fourier transform of $f(x, y)$. Considering the case when the $\mathrm{k}$-space is acquired as consecutive bands, as in the case of segmented EPI, PROPELLER, and the proposed method, one can neglect the inter-band motion. This is true because the entire band is acquired during a single read-out period. Thus, planar rigid motion parameters during the acquisition can be regarded as a function of the band number. A planar rigid motion is the combination of translational and rotational motions. It is well known that the rotation of an object about the center of the image domain results in the same rotation of its $\mathrm{k}$-space, while translational shift results in a linear phase term multiplied in the k-space. ${ }^{2}$ Thus the effect of the motion can be written as,

$$
F_{d}\left(k_{x}, k_{y}\right)=\exp \left[-j 2 \pi\left(\delta_{x} k_{x}+\delta_{y} k_{y}\right)\right] F_{\theta}\left(k_{x}, k_{y}\right)
$$

Here $F_{d}\left(k_{x}, k_{y}\right)$ is the motion-distorted MRI signal and $\delta_{x}, \delta_{y}$, and $\theta$ are the translation in the $x$-direction, the translation in the $y$-direction and the rotation angle, respectively, and $F_{\theta}\left(k_{x}, k_{y}\right)$ is defined as,

$$
F_{\theta}\left(k_{x}, k_{y}\right)=F\left(k_{x} \cos \theta+k_{y} \sin \theta,-k_{x} \sin \theta+k_{y} \cos \theta\right)
$$

The motion correction problem is that of estimating the unknown motion parameters $\delta_{x}, \delta_{y}$, and $\theta$ and using these parameters to reconstruct an artifact-free image. An illustration of this motion when a k-space band is acquired is shown in Figure 2.

Starting from the general assumption of rigid body motion, we consider the case when the acquisition of the k-space is in the form of overlapping bands of finite number of lines arranged in a rectilinear fashion to cover the k-space area of interest. We also assume that an averaging factor of at least two is desired (i.e., Number of excitation or NEX $\geq 2$ ), to make the inter-slice motion problem nontrivial. Instead of acquiring a full $\mathrm{k}$-space of each image and then averaging the result, we propose a new acquisition strategy based on acquiring the k-space in consecutive bands having (100.(NEX-1)/NEX)\% overlap going from one end of the phase encoding direction to the other end. For example, when NEX is 2, the overlap will be $50 \%$. Figure 3 illustrates the acquisition strategy proposed. Each band consists of a finite number of phase encoding lines acquired in a single

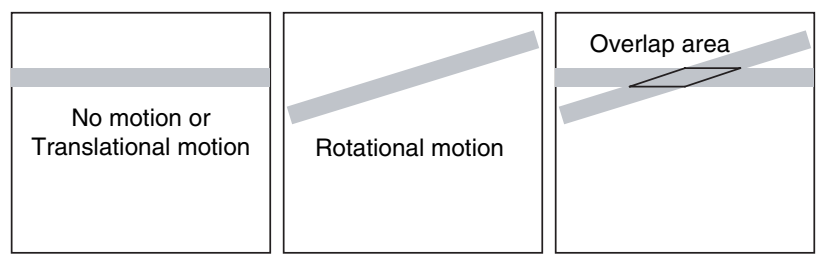

Fig. 2. Different cases in k-space band acquisition: no motion or translational motion (left), rotational motion while acquiring the same band (center). The overlap area between consecutive acquisitions in the worst case scenario is shown on the right.

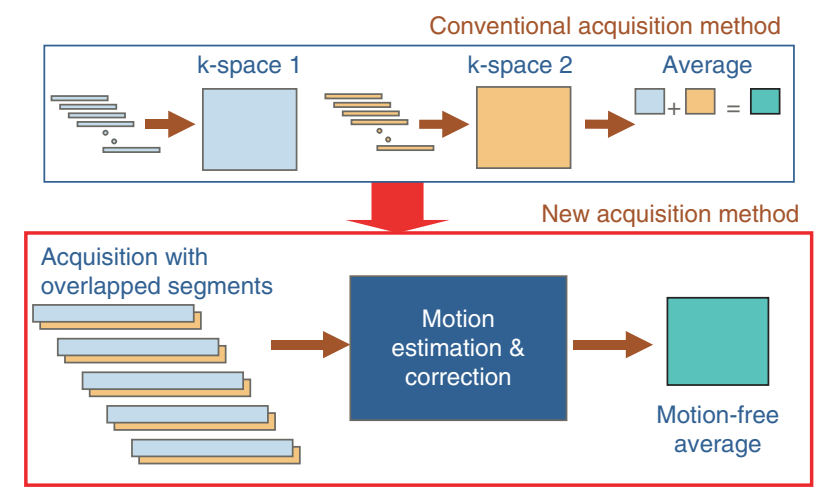

Fig. 3. Basic idea of the new method. Instead of doing the image acquisitions independently then performing the average, the acquisitions will be overlapped in such a way to allow the new overlap localization strategy to reconstruct an artifact-free image while maintaining the same acquisition time.

shot so that the inter-band motion effect is limited. In case of no motion, this overlap provides the additional acquisitions required by the selected NEX value, while in the case when inter-band motion occurs; the proposed overlap provides the information that enables the determination of motion parameters as a generalization of the floating navigator echo. ${ }^{2}$

The process of estimating the motion parameters is done in two steps: rotation estimation then 2-D translation estimation. From the geometry of the acquisition in the k-space in Figure 2, the presence of rotation amounts to varying the area of overlap between the two consecutive bands or blades. Hence, given that this geometry is known a priori, if we compute a similarity measure between the areas of overlap at each possible rotation angle within a predetermined range of possible angles, it is possible to determine the rotation angle as the one having the highest similarity measure. The utilized method is based on computing the correlation coefficient of the magnitudes of the points in the overlap areas from the two different acquisitions normalized by the number of points in the overlap area. This allows for the similarity measure to be independent of the size of the overlapping area. This is different from ${ }^{3}$ where only an arc navigator is used in that using the whole overlap area makes the overall SNR of the estimation better. Hence, there is an expected trade-off between accuracy and computational complexity in these two techniques. Once the rotation is determined, it is straightforward to determine

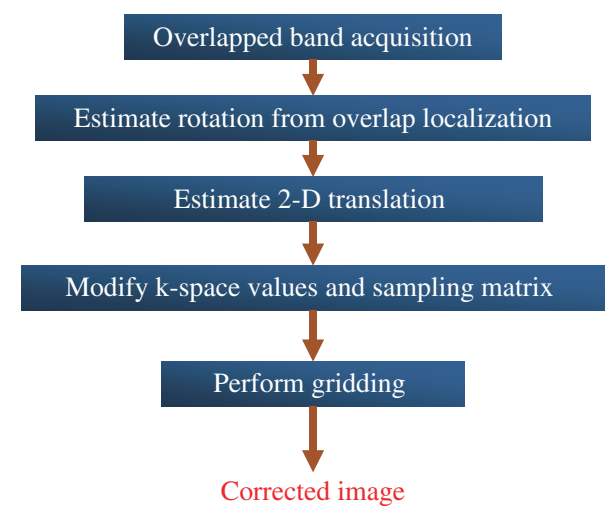

Fig. 4. Basic block diagram of the new method. 
the 2-D translation using the floating navigator echo technique. ${ }^{2}$ This process is done between each two consecutive bands with correction for translational motion for the most recently acquired band. On the other hand, the rotational motion can be corrected only during the reconstruction process given the sampling nonuniformity introduced into the k-space by this type of motion. The reconstruction includes an interpolation step to calculate the $\mathrm{k}$-space data on a rectilinear grid using gridding algorithms. ${ }^{4-5}$ A block diagram of the new technique is shown in Figure 4.

\section{RESULTS}

The proposed algorithm was verified using simulated motion on numerical phantoms as well as real human head images acquired from a normal human volunteer obtained on a Siemens Magnetom Trio 3T system. The volunteer was instructed to move during the data acquisition to induce motion artifacts. The acquisition parameters were as follows: imaging plane: sagittal, imaging sequence: fast spin echo, TR: $500 \mathrm{~ms}$, TE: $15 \mathrm{~ms}$, Matrix: $256 \times 256$, and ETL: 16 , NEX: 2 . Figure 5 shows an example of rotation estimation using the proposed method. The correlation coefficient is computed and for different overlap angles and the angle at which maximum correlation is found is used as the rotation estimate. In Figure 6, the results of applying the new method to numerically simulated axial brain slices are shown. Motion-corrupted images were generated from a single axial slice simulating random intra-slice and inter-slice motion artifacts as

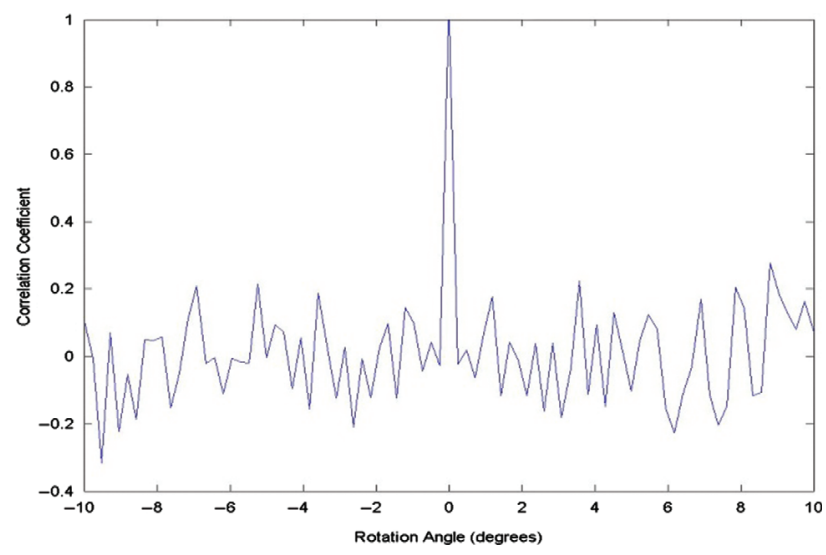

Fig. 5. Example of rotation estimation using the proposed method. The correlation coefficient is computed and for different overlap angles and the angle at which maximum correlation is found is used as the rotation estimate.
Motion-free

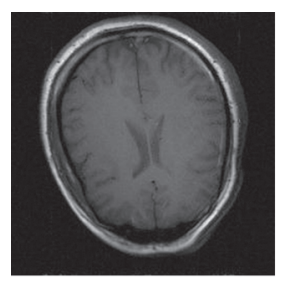

Motion-corrupted

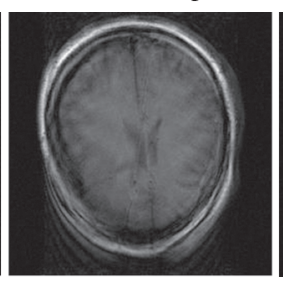

Corrected

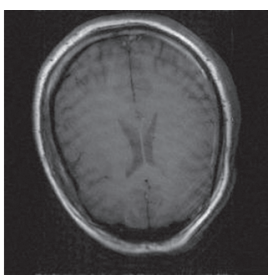

Fig. 6. Results of applying the new method to simulated data of axial brain slices of a human. As can be observed, substantial improvement is seen but ringing artifacts are visible in the corrected image due to possible k-space voids.

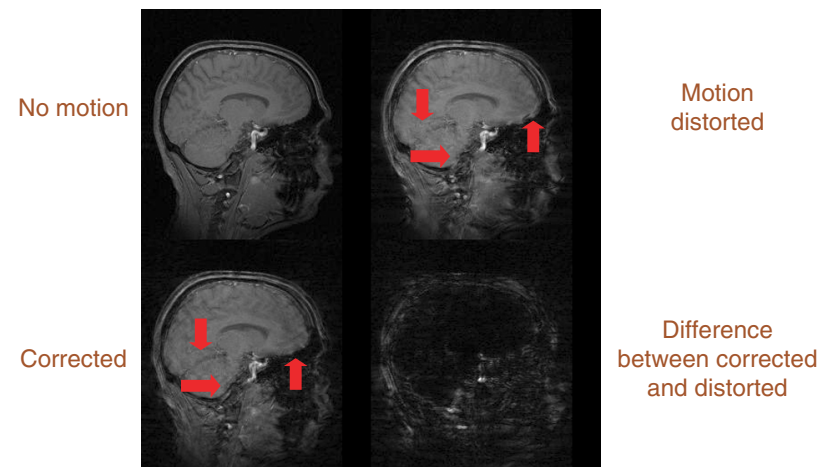

Fig. 7. Results of applying the new method to real data of sagittal brain slices of a human volunteer. Arrows point to areas in the image where correction is clearly visible. The difference image show the error removed by the new method.

shown in Figure 6(b). When the new method was applied to estimate and correct the simulated motion, a significant improvement in the reconstructed image was obtained as can be observed in Figure 6(c). A wide range of motion parameters was simulated to test the accuracy of the proposed method and simulation of noisy data was performed to test the robustness of the solution under different SNR conditions. The results indicate that the new method is capable of detecting rotations with a mean error as low as \pm 0.1 degrees (which is more accurate $\operatorname{than}^{3}$ ) and translation with an error that is always less than \pm 0.2 of the pixel width. Given that the similarity measures used implicitly average the data, the technique was found to be robust against noise in our experiments. Figure 7 shows the results obtained from the real data. The arrows show several locations where the improvement is most visible. As can be noticed, the correction substantially improves the resolution of the image. However, ringing artifacts appear more prominent in the corrected image due to the problem of k-space voids shown in Figure 8 (also previously reported in Ref. [10]). Further investigation is needed to address this problem from both acquisition and reconstruction perspectives.

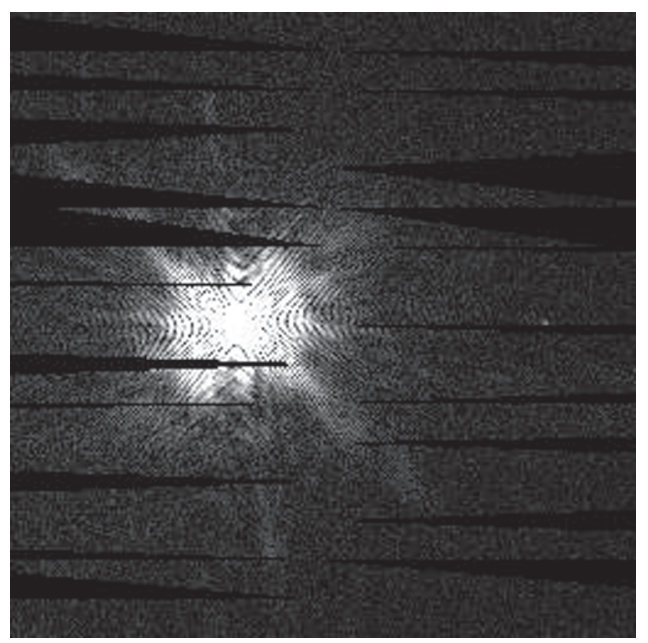

Fig. 8. Illustration of the problem of k-space voids where the rotated bands cause small scattered areas of the k-space to be missing thus leaving residual sampling artifacts in the reconstructed image after motion correction. 


\section{DISCUSSION}

In theory, the new method is comparable to the method in Ref. [6] and its faster variant in Ref. [25] when applied to data with intraslice motion only. While these methods estimate and correct the motion between segments in the spatial domain, the new method does that in the k-space. Given the orthogonality of the Fourier transformation, these two approaches are equivalent and should lead to the same results. Similar comparison can be seen between the new method and the AIR technique in Ref. [26] when dealing with inter-slice motion. So, as far as performance comparison with these techniques when faced with only intraslice motion or inter-slice motion, it is not possible to find an advantage to the new method. However, when faced with both types, the new method has an advantage in that it is formulated as a hybrid between these two methods and can deal with both motion types simultaneously.

The new method has a clear computational advantage over the leading postprocessing based motion correction method. ${ }^{6,25}$ Given the fact that the assumption of negligible motion within bands holds true in practical imaging settings, the new method delivers the same performance as ${ }^{6,25}$ with $\mathrm{O}\left(N^{2}\right)$ computations instead of $\mathrm{O}\left(N^{3} \log N\right)$ for these methods (nearly two orders of magnitude less computations). This is mainly due to the fact that the new method deals with much smaller registration problems and also because such registration is performed in the k-space rather than in the spatial domain. The new method combines the advantages of navigator echo based methods to simplify the process of motion estimation while using postprocessing techniques to estimate and correct the motion at no additional cost in acquisition time. Therefore, the new method has potential for practical use given its modest acquisition and computational requirements.

\section{CONCLUSIONS}

A new acquisition and processing strategy was proposed to suppress both intra-slice and inter-slice motion types more accurately based on the localization of overlapped k-space areas in between acquisitions. The new method was experimentally verified using simulated motion on numerical phantom as well as real images. The new technique has the advantage of not requiring additional acquisition time making its practical application possible.

\section{References and Notes}

1. R. L. Ehman and J. P. Felmlee, Radiology 173, 255 (1989).

2. Y. M. Kadah, A. Abaza, A. Fahmy, A. M. Youssef, K. Heberlein, and X. Hu, Magn. Reson. Med. 51, 403 (2004).

3. H. M. Ahmed, R. E. Gabr, A. M. Youssef, and Y. M. Kadah, Combined intraand inter-slice motion artifact suppression in magnetic resonance imaging, Proc. SPIE Medical Imaging 2003, San Diego, February (2003).

4. J. I. Jackson, C. H. Meyer, D. G. Nishimura, and A. Macovski, IEEE Trans. Med. Imag. 10, 473 (1991).

5. Y. M. Kadah, A. Fahmy, R. Gabr, K. Heberlein, and X. Hu, Int. J. Biomed. Imag. 2006, 1 (2006).

6. D. Atkinson, D. L. G. Hill, P. N. R. Stoyle, P. E. Summers, and S. F. Keevil, IEEE Trans. Med. Imag. 16, 903 (1997).

7. M. L. Wood and R. M. Henkelman, Med. Phys. 13, 794 (1986).

8. T. Mitsa, K. J. Parker, W. S. Smith, A. M. Tekalp, and J. Szumowski, IEEE Trans. Med. Imag. 9, 310 (1991).

9. M. Hedley, H. Yan, and D. Rosenfeld, IEEE Trans. Med. Imag. 10, 548 (1991).

10. C. Weerasinghe and $\mathrm{H}$. Yan, IEEE Trans. Med. Imag. 17, 310 (1998).

11. H. W. Korin, F. Farzaneh, R. C. Wright, and S. J. Riederer, Magn. Reson. Med. 12, 99 (1989).

12. R. A. Zoroofi, Y. Sato, S. Tamura, H. Naito, and L. Tang, IEEE Trans. Med. Imag. 14, 471 (1995).

13. L. Tang, M. Ohya, Y. Sato, S. Tamura, H. Naito, K. Harada, and T. Kozuka, Systems and Computers in Japan 26, 88 (1995).

14. M. Hedley, H. Yan, and D. Rosenfeld, IEEE Trans. Sig. Proc. 39, 1428 (1991).

15. M. Hedley, H. Yan, and D. Rosenfeld, IEEE Trans. Med. Imag. 10, 40 (1991).

16. M. Hedley and H. Yan, IEEE Trans. Med. Imag. 11, 233 (1992).

17. J. L. Durek and P. M. Pattany, Magn. Reson. Med. 7, 251 (1989)

18. B. Madore and R. M. Henkelman, Med. Phys. 23, 109 (1996).

19. J. P. Felmlee, R. L. Ehman, S. J. Riederer, and H. W. Korin, Radiology 179, 139 (1991).

20. P. M. Pattany, J. J. Phillips, L. C. Chiu, J. D. Lipcamon, J. L. Duerk, J. M. McNally, and Surya N. Mohapatra, J. Comput. Assist. Tomogr. 11, 369 (1987).

21. R. A. Zoroofi, Y. Sato, S. Tamura, and H. Naito, IEEE Trans. Med. Imag. 15, 768 (1996).

22. T. S. Sachs, C. H. Meyer, P. Irarrazabal, B. S. Hu, D. G. Nishimura, and A. Macovski, Magn. Reson. Med. 34, 412 (1995).

23. E. M. Haacke and G. W. Lenz, AJR 148, 1251 (1987).

24. X. Hu, T. H. Le, T. Parrish, and P. Erhard, Magn. Reson. Med. 34, 201 (1995).

25. A. Manduca, K. P. McGee, E. B. Welch, J. P. Felmlee, R. C. Grimm, and R. L. Ehman, Radiology 215, 904 (2000).

26. R. P. Woods, S. T. Grafton, C. J. Holmes, S. R. Cherry, and J. C. Mazziotta, Journal of Computer Assisted Tomography 22, 139 (1998). 